\title{
Contamination Des Eaux De Puits Par Les Salmonelles Et Les Vibrions non-O1/non-O139 Dans Les Quartiers Précaires Du Sixième Arrondissement De Cotonou (Sud-Bénin)
}

\author{
Hounsounou E.O. \\ Institut National de l'Eau (INE), \\ Université d'Abomey-Calavi (UAC), Cotonou, Bénin
}

Laboratoire des Normes et Contrôle de Qualité Microbiologique, Nutritionnelle et Pharmacologique (LNCQ $\left.{ }^{\mathrm{MNP}}\right)$, Faculté des Sciences et Techniques (FAST), Université d'Abomey-Calavi (UAC), Cotonou, Bénin

Chaire Internationale de Physiques, Mathématiques et Applications

(CIPMA), Université d'Abomey-Calavi (UAC), Cotonou, Bénin

\section{Ayi-Fanou L.}

Unité de Biochimie et Biologie Moléculaire, Laboratoire de Biologie moléculaire et Environnement, Faculté des Sciences et Techniques (FAST), Université d'Abomey-Calavi (UAC), Cotonou, Bénin

Ayéna A.C.

Laboratoire des Normes et Contrôle de Qualité Microbiologique, Nutritionnelle et Pharmacologique (LNCQ $\left.{ }^{\mathrm{MNP}}\right)$, Faculté des Sciences et Techniques (FAST), Université d'Abomey-Calavi (UAC), Cotonou, Bénin

\section{Agassounon Djikpo Tchibozo M.}

Laboratoire des Normes et Contrôle de Qualité Microbiologique, Nutritionnelle et Pharmacologique (LNCQ $\left.{ }^{\mathrm{MNP}}\right)$, Faculté des Sciences et Techniques (FAST), Université d'Abomey-Calavi (UAC), Cotonou, Bénin

\section{Mama D.}

Institut National de l'Eau (INE), Université d'Abomey-Calavi (UAC), Cotonou, Bénin

\section{Doi: 10.19044/esj.2018.v14n6p252 URL:http://dx.doi.org/10.19044/esj.2018.v14n6p252}

\begin{abstract}
The well waters are solicited for several uses in the households in the sixth district of Cotonou. In order to assess the contamination of these wells waters by some pathogenic germs, one hundred and twenty (120) samples were collected for four (4) seasonal campaigns (two rainy and two dry) at the rate of thirty (30) samples per season. The study has focused on the research of Salmonella and choleraic vibrios by using the referenced methods. The
\end{abstract}


analysis results indicate the presence of salmonella sp. in $73.33 \%$ (high rainy season) ; $56.67 \%$ (high dry season) ; $43.33 \%$ (small rainy season) and $40 \%$ (small dry season) of well water samples. The strains of vibrios isolated do not agglutinate with serum anti-O1 and anti-O139. The burden in these nonO1/non-O139 vibrios in the waters varies from <1 to $10 \mathrm{CFU} / 100 \mathrm{ml}$. This pathogenic germ is identified in most of the well water $(46.67 \%)$ during the small dry season. These two pathogenic bacteria that are found out of standards in the water of the wells analyzed can cause minor or severe infections to the riparian population consumers.

Keywords: Well water, Bacteriological contamination, Salmonella, nonO1/non-O139 Vibrios, Sanitary risks

\section{Résumé}

Les eaux de puits sont sollicitées pour plusieurs usages dans les ménages du sixième arrondissement de Cotonou. Dans le but d'évaluer la contamination de ces eaux de puits par certains germes pathogènes, cent vingt (120) échantillons d'eau des puits ont été prélevés pendant quatre (4) campagnes saisonnières (deux pluvieuses et deux sèches) à raison de trente (30) échantillons par saison. L'étude a porté sur la recherche des salmonelles et des vibrions cholériques en utilisant des méthodes référencées. Les résultats d'analyse indiquent la présence des salmonelles (Salmonella sp.) dans 73,33 $\%$ (grande saison pluvieuse) ; 56,67 \% (grande saison sèche) ; 43,33\% (petite saison pluvieuse) et $40 \%$ (petite saison sèche) des échantillons d'eau de puits. Les souches de vibrions isolées n'agglutinent pas avec les sérums anti-O1 et anti-O139. La charge en vibrions non-O1/non-O139 dans les eaux varie de $<1$ à $10 \mathrm{UFC} / 100 \mathrm{ml}$. Ce pathogène est dénombré dans la plupart des eaux de puits $(46,67 \%)$ pendant la petite saison sèche. Ces deux bactéries pathogènes qui se retrouvent hors normes dans les eaux de puits analysées peuvent provoquer des infections bénignes ou graves à la population riveraine consommatrice.

Mots-clés : Eau de puits, Contamination bactériologique, Salmonelles, Vibrions non-O1/non-O139, Risques sanitaires

\section{Introduction}

L'eau destinée à la consommation humaine et aux usages domestiques habituels doit être potable (OMS, 1997). L'usage de l'eau à des fins alimentaires ou hygiéniques nécessite une excellente qualité physicochimique et bactériologique (Kahoul et Touhami, 2014). L'Organisation Mondiale de la Santé (OMS) estime que $80 \%$ des maladies qui affectent la population mondiale sont directement associées à une mauvaise qualité de 
l'eau, à un assainissement insuffisant et à une hygiène défectueuse (OMS, 2004). Face à ces problèmes, la surveillance de la qualité de l'eau est devenue une priorité de santé publique dans le monde entier (Bras et al., 2007).

L'approvisionnement en eau constitue un problème très alarmant dans les quartiers précaires des villes des pays en développement (Dongo al., 2008). Ceci est imputé à la forte proportion des habitants vivant dans les zones déshéritées au sein des populations urbaines en pleine expansion (OMS et UNICEF, 2007). A l'instar de nombreuses villes africaines, la capitale économique du Bénin appelée Cotonou a connu une extension rapide donnant lieu à des quartiers mis en place et occupés de façon anarchique avec l'augmentation de l'insalubrité et de la pollution environnementale (Capo, 2008). Pour cela, l'accès à l'eau potable fait partie des difficultés quotidiennes des ménages à faibles revenus des quartiers défavorisés (Odoulami, 2009 ; Hounsounou et $a l ., 2016^{\mathrm{a}}$ ). Ces ménages n'ont recours qu'aux sources d'eau issues des puits non protégés lesquelles sont peu satisfaisantes sur le plan hygiénique pour les usages domestiques (Odoulami, 2009). Cette catégorie de population est plus exposée aux maladies liées aux eaux de mauvaise qualité, à l'assainissement précaire et à la mauvaise hygiène (Sy et al., 2011). Les problèmes de santé liés à l'eau résultent essentiellement d'une contamination microbiologique (Aubry et Gaüzère, 2012). Les maladies causées par les microorganismes pathogènes comme les salmonelloses provoquées par Salmonella enterica et Salmonella bongori sont souvent de nature gastrointestinale (Blais et al., 2015). L'incidence de ces infections est de 16 millions de cas, avec 600000 décès par an dans le monde (Aubry, 2013). De même à Cotonou, des cas de salmonelloses sont régulièrement enregistrés (MSP, 2008-2012). Par ailleurs, le choléra constitue une autre maladie infectieuse dont 1,3 à 4,0 millions de cas surviennent chaque année dans le monde dont 21000 à 143000 de décès (Ali, 2015). A l'instar d'autres villes africaines, les épidémies de choléra sont fréquentes à Cotonou (Makoutodé et al., 2010 ; MSP, 2008-2012). Ainsi, 210 cas avec 4 décès, 88 cas dont un (1) décès, 402 cas dont un (1) décès et 346 cas avec 5 décès ont été enregistrés respectivement en l'an 2005, 2006, 2008 et 2016 à Cotonou.

Face aux multiples facteurs de risque de pollution microbiologique qui existent dans les quartiers précaires (Hounsounou et $a l ., 2016^{\mathrm{a}}$ ), couplés à l'influence de la fluctuation climatique sur la qualité des eaux (Benajiba et al., 2013), la présente étude vise à évaluer l'état de contamination saisonnière des eaux de puits à usages domestiques par les salmonelles et les vibrions cholériques dans les quartiers précaires du sixième arrondissement de Cotonou. 


\section{Matériel et méthodes}

\section{Localisation de la zone d'étude}

Situé dans la zone périphérique de la Commune de Cotonou, le sixième arrondissement de Cotonou est limité au nord par le lac Nokoué, au sud par le cinquième arrondissement, à l'est par la lagune de Cotonou, à l'ouest par les septième, huitième et neuvième arrondissements de Cotonou (figure1). Le secteur d'étude est le deuxième arrondissement le plus peuplé des treize que compte la Commune de Cotonou où les problèmes d'hygiène et d'assainissement de base (gestion des déchets ; défécation en plein air, etc.) sont dominants (Hounsounou et $a l ., 2016^{\mathrm{b}}$ ). Le climat est de type équatorial avec une alternance de deux saisons pluvieuses et de deux saisons sèches. Pendant la grande saison des pluies, l'arrondissement est menacé par des inondations avec un faible écoulement de l'eau pluviale et un mauvais drainage des eaux usées. Le relief est plat avec le niveau de la nappe phréatique variant entre 0 et $5 \mathrm{~m}$. La platitude du relief et l'hydrogéologie (vitesse d'infiltration, perméabilité du sol et le faible niveau de la nappe) de la ville de Cotonou facilitent la recharge de la nappe phréatique exploitée par les ménages par le biais des puits traditionnels (Odoulami, 2009).

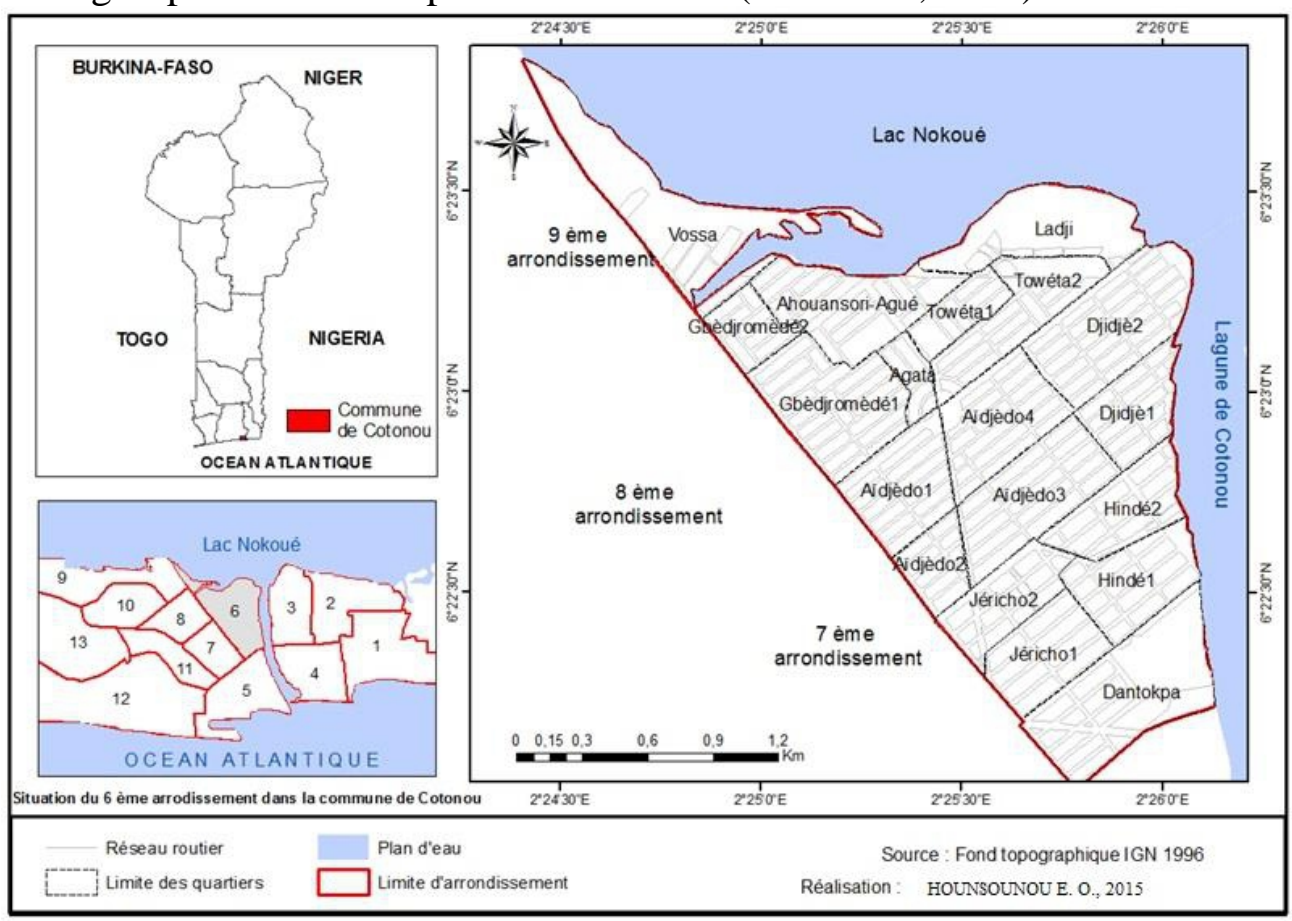

Figure 1 : Carte de localisation géographique de la zone d'étude

\section{Echantillonnage et modalités de prélèvement des eaux de puits}

Cent vingt (120) échantillons d'eau de puits ont été prélevés au cours de quatre (04) campagnes saisonnières couvrant une grande saison pluvieuse 
(juin 2015), une grande saison sèche (février 2016), une petite saison pluvieuse (octobre 2016) et une petite saison sèche (août 2017) à raison de trente (30) échantillons par saison. Les puits ont été sélectionnés suivant des critères tels que l'accessibilité du puits, la fréquentation et le consentement du propriétaire. Les puits sont localisés dans les quartiers retenus (Vossa, Ahouansori-Agata, Ahouansori-Towéta (I et II), Ladji, Hindé (I et II) et Djidjè (I et II)). Ces quartiers ont été choisis en fonction de la situation précaire des ménages qui utilisent ces eaux de puits pour leurs besoins domestiques (Hounsounou et al., 2014).

Le prélèvement des échantillons a été fait en duplicata par la méthode de Rodier et al. (2009), adaptée aux eaux souterraines moins profondes à raison de 1 litre dans une bouteille en matière polyéthylène. Ces échantillons sont conservés dans une glacière munie de carboglace et acheminés directement au laboratoire. Chaque échantillon a été identifié au moyen d'une fiche de prélèvement référencée (date, lieu, numéro, heure du prélèvement). Les codes des échantillons sont formulés à partir de la lettre $\mathrm{P}$ : (Puits) suivie de l'initial du nom des quartiers et des numéros d'ordre. Les coordonnées géographiques des points de prélèvement ont été prises à l'aide d'un GPS de marque CARMIN pour la réalisation des cartes de présence ou absence des salmonelles.

\section{Recherche des salmonelles}

Les salmonelles ont été recherchées par la méthode NF EN ISO 6579/A1 (2007) qui a nécessité quatre (4) étapes standardisées. Il s'agit d'un préenrichissement, d'un enrichissement, d'un isolement et d'une identification (Rodier et al., 2009).

Au cours du préenrichissement, $25 \mathrm{ml}$ de l'échantillon d'eau de puits ont été filtrés sur une membrane de cellulose stérile de porosité de $0,45 \mu \mathrm{m}$. $100 \mathrm{ml}$ de l'échantillon ont été considérés à raison de $25 \mathrm{ml}$ par test. Après filtration, la membrane a été ensemencée avec $250 \mathrm{ml}$ de l'eau peptonnée tamponnée contenue dans un erlenmeyer. La solution a été incubée à $37^{\circ} \mathrm{C}$ pendant 24 heures.

L'enrichissement a été réalisé avec le Bouillon au Sélénite Cystine (BSC) et le Bouillon Rappaport Vassiliadis (RV10) complet. $20 \mathrm{ml}$ de chacun de ces bouillons ont été versés dans un tube. Puis, $1 \mathrm{ml}$ de la solution préenrichie a été introduit dans chacun de ces tubes de bouillons qui ont été incubés à $37^{\circ} \mathrm{C}$ pendant 48 heures.

Ensuite, l'isolement des salmonelles a été fait par öse à partir des tubes d'enrichissement avec les milieux gélosés Hektoen et Salmonella/Shigella, puis de nouveau incubés à $37{ }^{\circ} \mathrm{C}$ pendant 24 heures. Sur la gélose Hektoen, les présumées colonies de salmonelles ont présenté une coloration bleue ou 
verte à centre noir tandis que sur la gélose Salmonella-Shigella, les colonies sont incolores transparentes et incolores à centre noir.

Avant l'étape d'identification biochimique, les colonies typiques ou suspectes ont été selectionnées sur les géloses d'isolements ; puis cinq (05) colonies caractéristiques ont été repiquées chacune sur le milieu gélosé Kliger et sur la gélose nutritive ordinaire pour vérification de leur pureté. En effet, l'ensemencement sur le milieu Kliger a été fait par piqûre du culot et par strie longitudinale sur la pente et par stries sur la gélose nutritive. Il a été considéré les résultats qui ont montré un culot jaune du milieu de Kliger (fermentation $\mathrm{du}$ glucose) et une pente rouge (absence de fermentation du lactose). L'incubation a été faite à $37{ }^{\circ} \mathrm{C}$ pendant 24 heures. Ensuite, les tests de 1'oxydase, de coloration de Gram ont été effectués avec la culture de la gélose nutritive en considérant les (glucose + et lactose -) obtenues avec la gélose kliger. Enfin, la recherche de l'uréase et de l'indole a été faite à partir de la culture du milieu kliger sur le milieu urée-indole (uréase- ; indole-). La recherche de la lysine décarboxylase (LDC) été faite sur le milieu XLD. Les résultats positifs sont oxydase -, Gram- ; uréase - ; indole- et LDC+. Les résultats de chaque échantillon d'eau de puits analysé ont été exprimés en terme de présence ou absence de salmonelle dans $100 \mathrm{ml}$ d'eau.

\section{Recherche et numération de vibrions cholériques}

La recherche et la numération de vibrions cholériques ont été effectuées suivant les méthodes qualitative et quantitative de Sudre et LeGoff (2011). Deux lots d'échantillons ont été réalisés aspetiquement au laboratoire. Au cours de la méthode qualitative (préenrichissement), à partir du premier lot d'échantillon, 1 litre a été filtré sur des membranes filtrantes de porosité 0,22 $\mu \mathrm{m}$. Cinq (5) membranes ont été utilisées pour la filtration de 1 litre à raison de $200 \mathrm{ml}$ par membrane. Après filtration, chacune des membranes a été ensemencée dans $50 \mathrm{ml}$ d'eau peptonnée alcaline contenue dans un tube ; l'ensemble a été agité puis incubé pendant 16 heures à $30^{\circ} \mathrm{C}$. Les tubes de préenrichissement ont été sortis de l'incubateur sans être agités car les vibrions se situent en surface dans le surnageant qui constitue un film blanchâtre à la surface de la colonne de liquide. Pour l'isolement, le surnageant du tube de préenrichissement a été prélevé par öse puis ensemencé par stries sur une boîte de gélose sélective thiosulfate-citrate-bile-saccharose (TCBS). Cette boîte a été incubée à $37^{\circ} \mathrm{C}$ pendant 24 heures. Après la culture, les colonies plates, lisses et de couleur jaune ont été considérées comme les colonies suspectes. En absence d'un bon isolement des colonies, un nouvel isolement sur la gélose TCBS s'effectue. Au cours de la confirmation, trois (03) colonies suspectes ont été repiquées sur la gélose nutritive alcaline pour identification. L'incubation a été faite pendant 16 heures à 24 heures à $37^{\circ} \mathrm{C}$. Après l'incubation, le test de l'oxydase et la coloration de Gram ont été 
réalisés. Si les tests d'oxydase et de coloration de Gram sont concluants (oxydase + , Gram-), le test d'agglutination a été réalisé à l'aide des sérums anti-O1 et anti-O139 pour la vérification de l'appartenance des vibrions identifiés aux sérogroupes (O1 ou 139), seuls sérogroupes responsables du choléra chez l'homme.

La méthode quantitative, considérée comme test confirmatif et quantitatif a permis le dénombrement des vibrions. En effet, l'analyse quantitative a été réalisée sur le deuxième lot d'échantillon (préalablement conservé). Comme dans le cas de la méthode qualitative, 1 litre d'échantillon a été filtré avec cinq (5) membranes à raison de $200 \mathrm{ml}$ par membrane de porosité $0,22 \mu \mathrm{m}$. Chaque membrane a été mise en culture indépendamment sur des boîtes de gélose TCBS. L'incubation a été faite à $37^{\circ} \mathrm{C}$ pendant 24 heures. Les colonies suspectes sont jaunes et plates, avec un halo. Après l'incubation, Le dénombrement des colonies suspectes sur chaque boîte a été fait. Pour la confirmation, les colonies suspectes ont été repiquées sur la gélose nutritive alcaline. L'incubation a été faite pendant 24 heures à $37^{\circ} \mathrm{C}$. Le test de l'oxydase et la coloration de Gram ont été effectués après l'incubation. Si lesdits tests sont concluants, le test d'agglutination s'effectue à l'aide des sérums anti-O1 et anti-O139. A partir du dénombrement préalablement fait le résultat a été exprimé en nombre de colonies pour $100 \mathrm{ml}(\mathrm{UFC} / 100 \mathrm{ml})$ et a été comparé aux critères de la réglementation béninoise qui est de 0 UFC/100 $\mathrm{ml}$ d'eau de puits.

\section{Analyse statistique}

Les résultats relatifs à la recherche de salmonelles ont été exprimés en termes de présence ou absence dans les eaux. Des cartes de leur répartition sur la zone d'étude ont été réalisées à l'aide des coordonnées géographiques relevées au niveau de chaque site de prélèvement. Une analyse statistique descriptive (moyenne, minimum et maximum) des données issues du dénombrement de vibrions a été effectuée à partir du logiciel Statistica version 6. Ensuite le tableur Excel a servi à la réalisation d'un graphe montrant la variation saisonnière des données.

\section{Résultats}

Les résultats de la recherche des salmonelles et des vibrions chlolériques varient selon les différentes campagnes saisonnières.

\section{Résultats issus de la recherche de Salmonella}

Il ressort des résultats d'analyse que les échantillons d'eau de puits analysés contiennent des salmonelles (Salmonella sp.) En effet, la présence de ce germe est notée dans les eaux de puits pendant la grande saison pluvieuse $(73,33 \%)$; la grande saison sèche $(56,67 \%)$, la petite saison pluvieuse $(43,33$ 
$\%)$ et la petite saison sèche (40 \%). Le plus fort taux de contamination $(73,33$

$\%$ ) est observé au cours de la grande saison pluvieuse et le plus faible en petite saison sèche $(40 \%)$. Les puits affectés par cette contamination microbienne se répartissent diversement sur les puits des différents quartiers selon les campagnes saisonnières (figures 2 à 5). Ainsi, au cours de la grande saison pluvieuse, les proportions d'échantillons d'eau ayant révélé la présence de ce germe sont de $40 \%$ à Vossa et Agata; $60 \%$ à Towéta et $100 \%$ à Ladji, Hindé et Djidjè. En grande saison sèche la proportion d'eau de puits contenant les salmonelles est de $40 \%$ à Agata, et Towéta; $60 \%$ à Vossa, Ladji, Djidjè et $80 \%$ à Hindé. Pour la petite saison pluvieuse, le taux de présence de salmonelles est de $20 \%$ à Hindé ; $40 \%$ à Vossa ; $80 \%$ à Agata et $100 \%$ à Djidjè . Quant à la petite saison sèche, $10 \%$ des échantillons révèlent la présence de Salmonella sp. dans les quartiers Ladji, Hindé, et Djidjè ; $40 \%$ à Agata ; $60 \%$ à Towéta et $100 \%$ à Vossa.

Par rapport à la fréquence de présence de salmonelles dans chaque puits, ce pathogène est détecté dans $100 \%$ des échantillons pendant au moins une (1) campagne saisonnière sur les (04) quatre. Les puits codés PV4 et PD3, situés respectivement à Vossa et à Djidjè présentent la forte fréquence de contamination (4/4) soit $100 \%$ des campagnes.

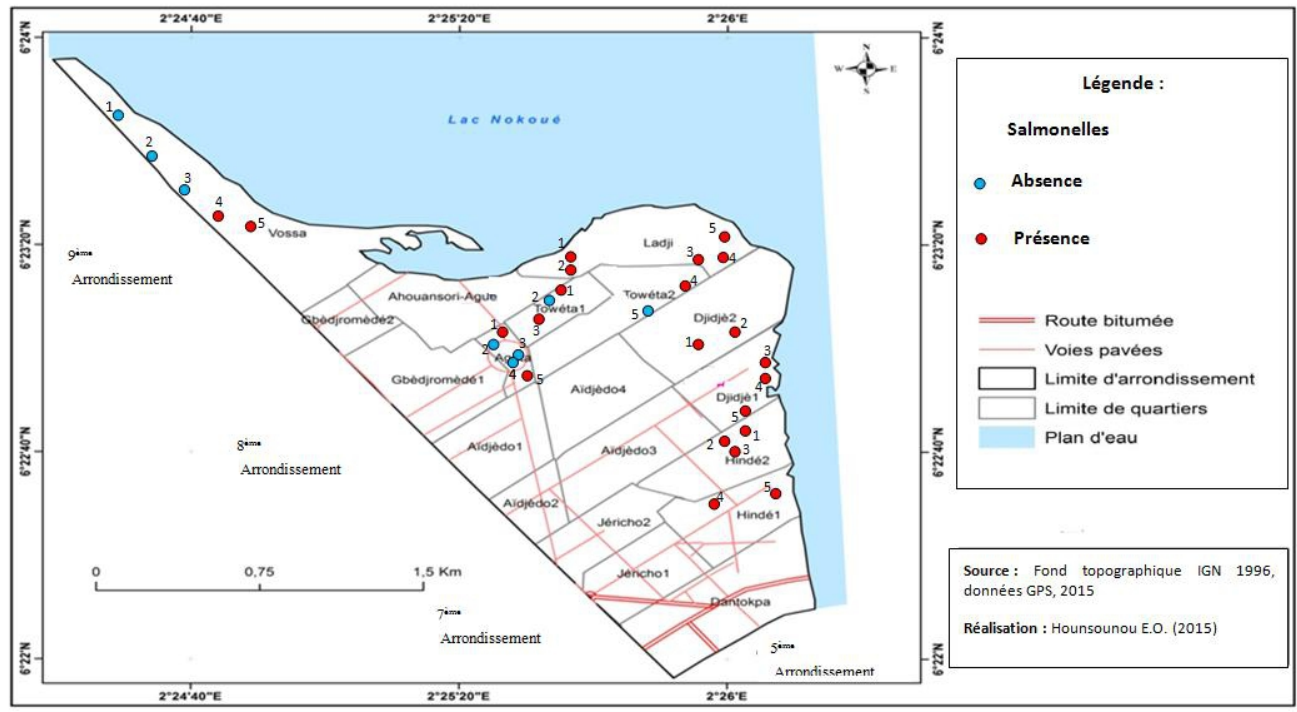

Figure 2 : Répartition de l'état de présence ou d'absence des salmonelles dans les eaux de puits analysées en grande saison pluvieuse 


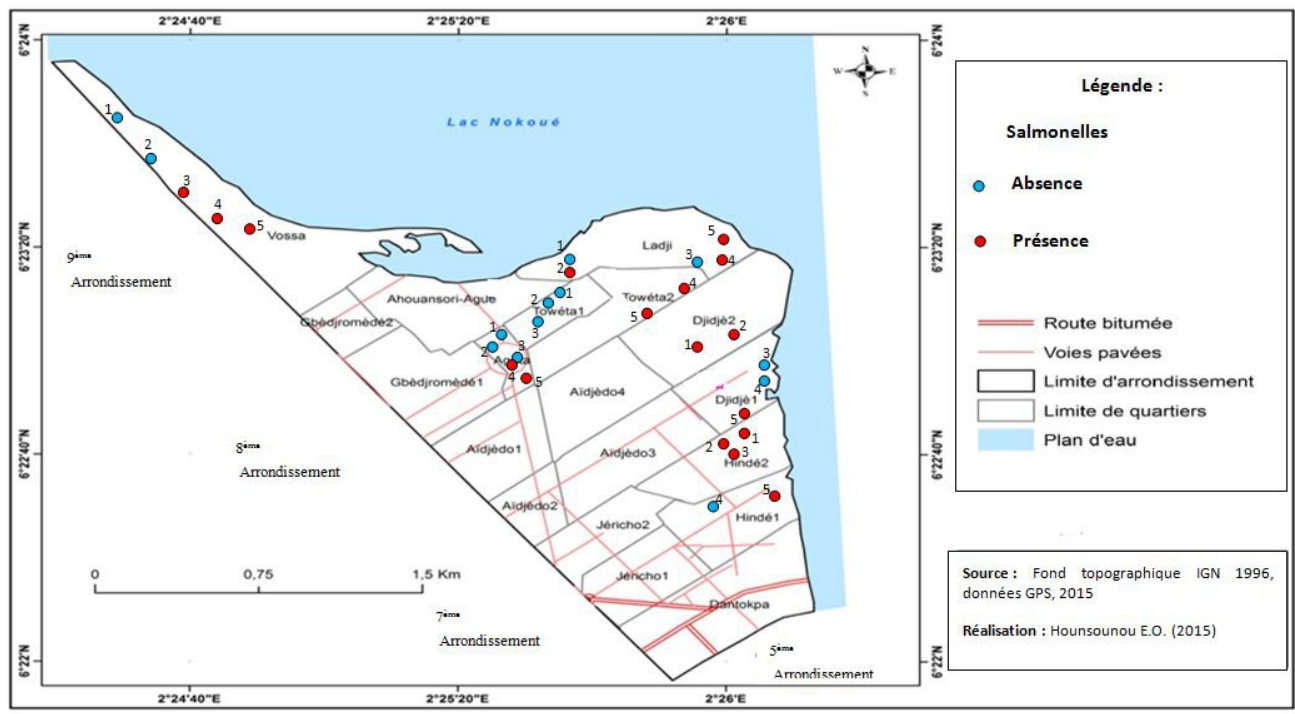

Figure 3 : Répartition de l'état de présence ou d'absence des salmonelles dans les eaux de puits analysées en grande saison sèche

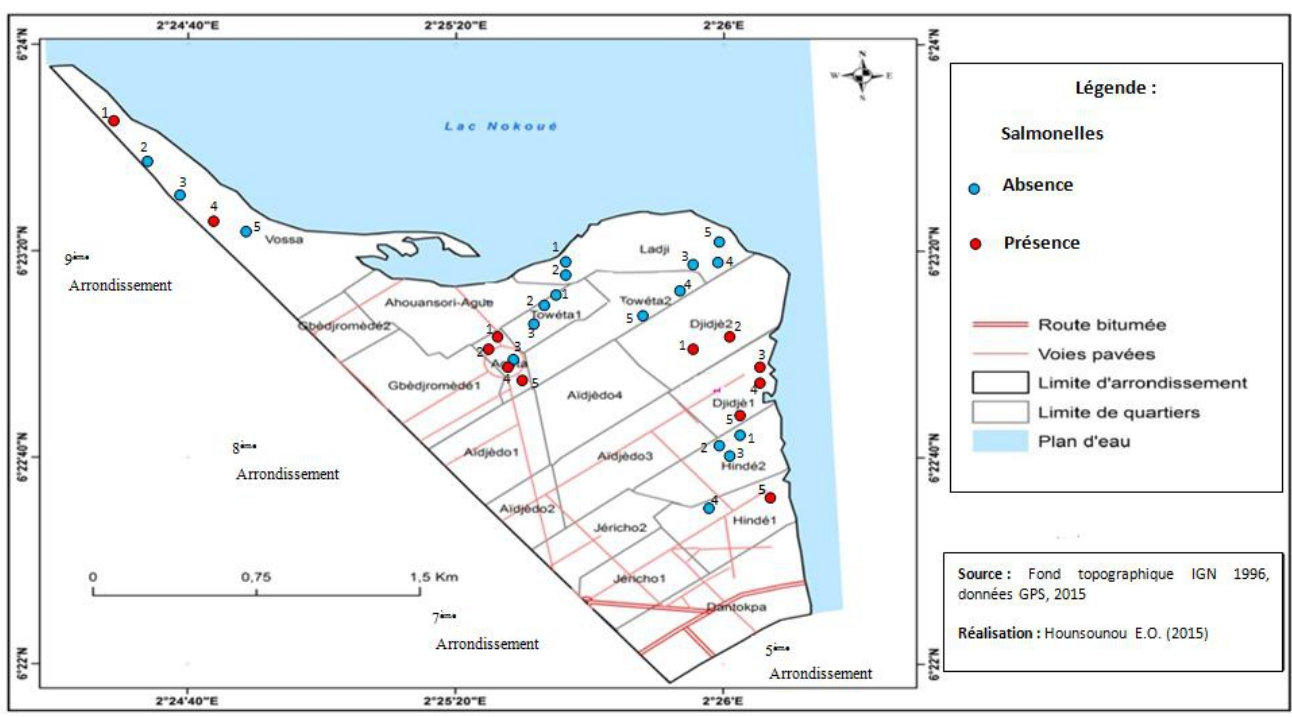

Figure 4 : Répartition de l'état de présence ou d'absence des salmonelles dans les eaux de puits analysées en petite saison pluvieuse 


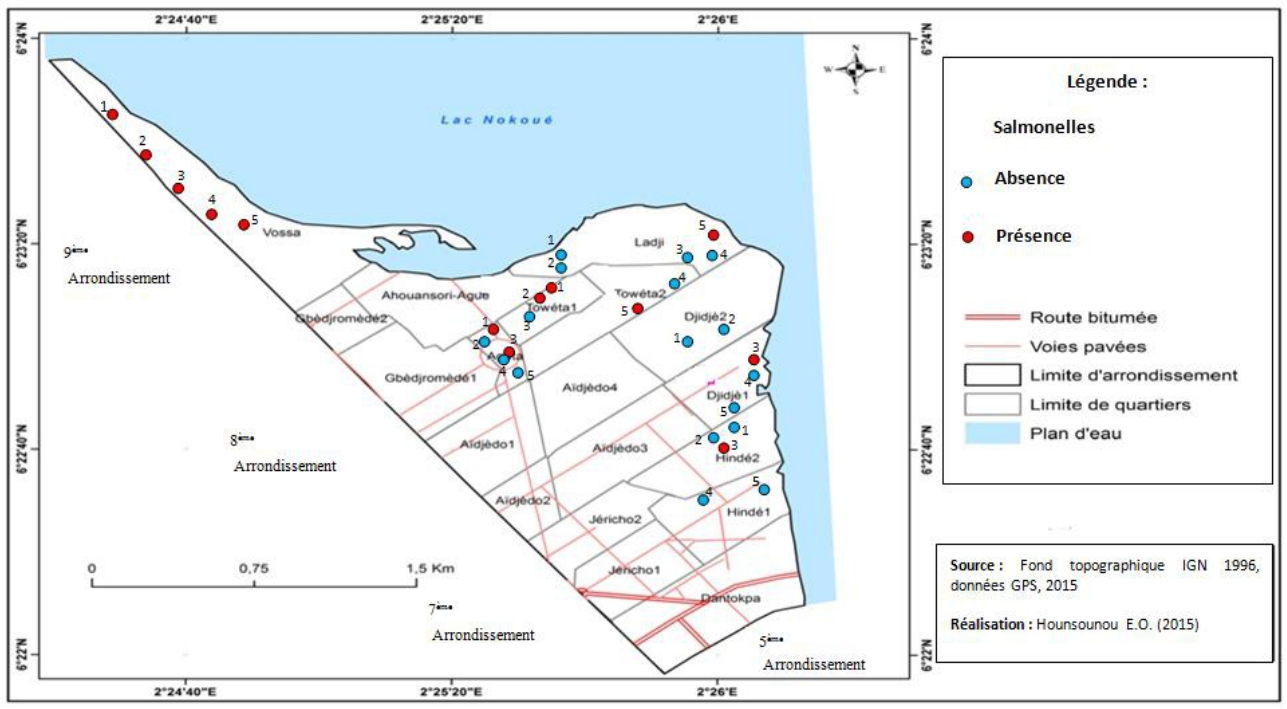

Figure 5 : Répartition de l'état de présence ou d'absence des salmonelles dans les eaux de puits analysées en petite saison sèche

\section{Résultats issus de la recherche de Vibrio cholerae}

L'analyse des résultats d'évaluation de la qualité des échantillons d'eau de puits révèle que la charge en vibrions non-O1/non-O139 est comprise entre $<1 \mathrm{UFC} / 100 \mathrm{ml}$ et $10 \mathrm{UFC} / 100 \mathrm{ml}$ (figure 6). La charge maximale est obtenue pendant les saisons pluvieuses dans les eaux de puits codés PV4, PT4 et PL3 situés respectivement dans les quartiers de Vossa, de Towéta et de Ladji. $10 \%$ à $16,67 \%$ des échantillons présentent ce germe pendant les saisons pluvieuses (figure 6). Cependant, le nombre le plus élevé (14 soit 46,67 $\%)$ de puits contenant les vibrions non-O1/non-O139 est observé au cours de petite saison sèche. Aucun des échantillons d'eau ne présente de vibrions pendant la grande saison sèche. Par ailleurs, par rapport à la fréquence d'exposition de chaque puits, la présence des vibrions non-O1/non-O139, est observée dans $50 \%$ des échantillons au cours d'une seule campagne saisonnière. Les puits codés PL3 et PL5, tous deux situés à Ladji ont les fortes fréquences d'exposition à ce vibrion qui sont respectivement de (2/4) et (3/4) soit $50 \%$ et $75 \%$ du nombre de campagnes réalisées. 


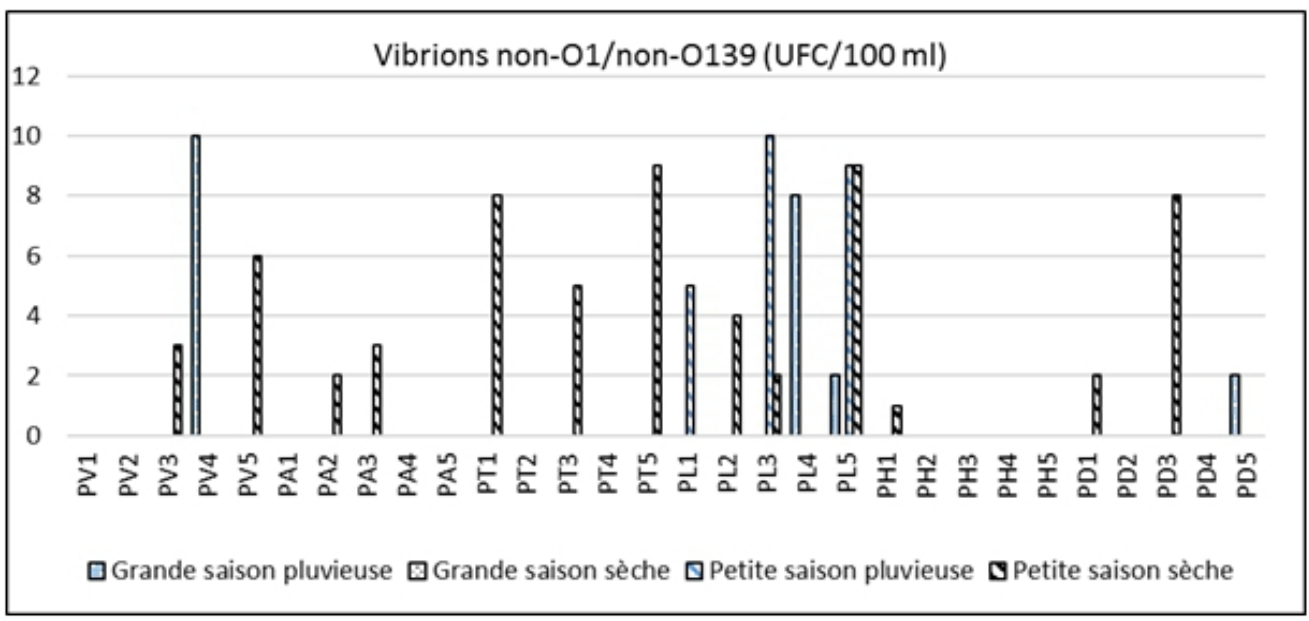

Figure 6 : Variation saisonnière de la charge en en vibrions non-O1/non-O139 des eaux de puits analysées

\section{Discussion}

Les résultats de l'analyse bactériologique des eaux de puits à usages domestiques prélevées dans les quartiers précaires du sixième arrondissement de Cotonou indiquent la présence de germes pathogènes tels que les salmonelles et les vibrions non-O1/non-O139. Le taux de présence des salmonelles (Salmonella sp.) des eaux varie en fonction des campagnes saisonnières. En effet, la présence des salmonelles dans plus de $40 \%$ des échantillons d'eau pendant les quatre campagnes est non conforme à la réglementation béninoise du 20 février 2001 qui recommande leur absence dans $100 \mathrm{ml}$ d'eau analysée. Cette présence pendant toutes les campagnes saisonnières indique que le problème de contamination lié à ce germe est continu. Les déjections humaines et animales constituent la principale source de pollution par les salmonelles car les hommes et les animaux les éliminent par les selles non seulement en cas de maladie mais aussi en tant que porteurs asymptomatiques (Rodier et al., 2009). Il ressort de cette étude que la présence des salmonelles dans les eaux de puits analysées peut être due aux déjections humaines et animales infectées parce que les travaux de Hounsounou et $a l .\left(2016^{\mathrm{b}}\right)$ dans la même zone d'étude ont révélé que $45 \%$ des concessions pratiquent fréquemment la défécation dans la nature. Il a été rapporté que les latrines, les dépotoirs de déchets et les puisards sont situés dans l'environnement immédiat (moins de $15 \mathrm{~m}$ ) des puits. Dans ces conditions, la contamination des eaux de puits par les déjections rejetées est favorisée par le ruissellement et l'infiltration des eaux pluviales (Haijoubi et al., 2017). Nos précédentes études (Hounsounou et al., 2017) dans cette zone, soulignaient que $20,8 \%$ des puisettes utilisées pour le prélèvement des eaux sont déposées à même le sol. De même, 93,33\% des puits ne sont pas couverts 
et non aménagés. Toutes ces raisons expliquent le fort taux $(73,33 \%)$ de présence de salmonelles observé pendant la grande saison pluvieuse. Par ailleurs, les travaux de Hounsounou et $a l .\left(2016^{\mathrm{b}}\right)$ avaient montré la pollution fécale des eaux de puits du sixième arrondissement de Cotonou en saison pluvieuse où il a été enregistré 43,34 \% de puits contaminés par Escherichia coli, germe indicateur de contamination fécale. Cela confirme davantage l'origine fécale des salmonelles retrouvées dans les eaux de puits étudiées. De même, les études de Degbey et al. (2011) et Dovonou et al. (2011) ont montré la présence couplée de Salmonella sp. et des germes fécaux dans les eaux de puits. Le risque de contamination des eaux de puits par les eaux usées domestiques existe (Rodier et al., 2009). L'ensemble des ces résultats confirme le bien fondé des études qui avaient rapporté qu'un nombre élevé de germes fécaux s'accompagne de pathogènes (Bourgeois et Leveau, 1991; Agassounon Djikpo Tchibozo et al., 2001).

Par rapport à la numération des vibrions cholériques dans les eaux de puits, il ressort la présence des vibrions non-O1/non-O139 qui constituent une espèce bactérienne qui n'est pas responsable d'épidémie de choléra (Quilici, 2011). Seules les espèces de Vibrio cholerae $\mathrm{O} 1$ et $\mathrm{O} 139$ sont incriminées dans les cas d'épidémiologie. Cependant, les vibrions non cholériques sont responsables des gastro-entérites (Fournier et Quilici, 2002). La présence de cette souche de vibrions dans les eaux de puits analysées peut être attribuée d'une part à l'apport des eaux de surface contaminées, et d'autre part à celles des estuaires contaminées ou non (Hernandez, 1989). Il s'agit notamment du lac Nokoué et de la lagune de Cotonou qui bordent la zone d'étude. Dovonou et $a l$. (2011) ont révélé que la contamination du lac Nokoué a d'incidence sur la qualité des eaux de puits environnants. La proximité de la lagune de Cotonou, en communication avec l'océan atlantique constitue aussi un facteur non négligeable de l'apport de vibrions non-O1/non-O139. La présence de ce germe a été identifiée dans les eaux des lagunes palavasiennes dans la région de Montpellier et dans les eaux côtières françaises de l'Atlantique. Les vibrions non-O1/non-O139 présentent une grande adaptation aux différents écosystèmes aquatiques. La variation saisonnière de leur charge dans les eaux de puits s'explique par l'influence des facteurs climatiques tels que les précipitations et la température (CHOLCLIM, 2006). En effet, la forte charge (10UFC/100 ml) de ces vibrions dans les eaux des puits codés PV4, PT4 et PL3 pendant la grande saison pluvieuse est due aux effets des inondations que subissent régulièrement les quartiers (Vossa, Towéta, Ladji) dans lesquels ils sont localisés (Odoulami, 2009). Par contre, le taux élevé (46,67 \%) de puits contenant les vibrions non-O1/non-O139 enregistré au cours de la petite saison sèche est lié à la température qui règne pendant cette période couplée à l'humidité issue de la saison des pluies précédentes. Selon Colwell et Huq (1994), il est plus fréquent d'isoler cette bactérie durant la période chaude. Par 
ailleurs, la présence des vibrions non-O1/non-O139 dans les eaux de puits étudiés peut aussi être liée aux conditions d'hygiène précaire notamment la défécation dans la nature et la proximité des latrines (Rodier et al., 2009). Cette affirmation est liée au fait que les travaux de Carter et al. (1981) ont rapporté que ce germe a été isolé dans les selles de sujets atteints de maladies diarrhéiques en Asie (Bangladesh, Hong Kong, Inde, Malaisie) et en Afrique (Afrique du Sud, Soudan). L'absence des vibrions cholériques dans l'ensemble des puits analysés peut s'expliquer par leur nombre relativement très faible dans ces eaux car leur recherche est plus aisée dans les selles à cause de leur abondance dans ce milieu (Quilici, 2011). De même cette absence peut être liée à l'état de stress de certains pathogènes dans les eaux. Ces derniers sont dits «viables mais non cultivables » (Gruzdev et al., 2011). La perte de culture est le résultat de divers stress (stress nutritionnel, thermique, lumineux) que subissent les bactéries fécales lorsqu'elles sont rejetées dans un milieu aquatique naturel (Roszak et Colwell, 1987 ; Gruzdev et al., 2011). Il est à noter que, ces bactéries viables non cultivables peuvent conserver leur pathogénicité (Habimana et al., 2014). Cela explique la fréquence des épidémies de choléra dans le secteur d'étude malgré la non détection de vibrions cholériques dans les eaux de puits. Selon Hernandez (1989), les vibrions non $\mathrm{O} 1$ sont plus fréquemment isolés du milieu aquatique que ceux provoquant le choléra.

Les bactéries pathogènes tels que les vibrions non-O1/non-O139 et les salmonelles sont généralement transmis aux humains par l'ingestion d'eau contaminée et sont responsables de diverses maladies (Momba et al., 2006). Alors, la consommation des eaux de puits ayant révélé ces germes constitue un facteur de risque sanitaire pour la population (Momba et al., 2006 ; Degbey et al., 2011 ). En effet, Salmonella plus précisément les deux espèces Salmonella enterica et $S$. bongori sont responsables des salmonelloses. Il s'agit entre autres de la fièvre typhoïde, des fièvres paratyphoïdes et des salmonelloses non typhoïdiques. Ils entraînent le plus souvent des gastroentérites (Aubry, 2013). Par rapport aux risques sanitaires liés aux vibrions non-O1/non-O139, ils sont moins virulents que ceux qui causent le choléra mais demeurent pathogènes (Hervio-Heath et al., 2002). Leur pathogénicité est à l'origine des infections sporadiques de gastro-entérites, des diarrhées et des abcès ou septicémies chez l'homme (Fournier et Quilici, 2002 ; Hervio-Heath et al., 2002 ; Dutta et al., 2013). Ces infections peuvent être gravissimes (Fournier et Quilici, 2002). L'ensemble de risques sanitaires liés à la consommation des eaux contenant les salmonelles et les vibrions nonO1/non-O139 justifient la prévalence de diarrhées et d'affections gastrointestinales rapportées par Hounsounou et $a l .\left(2016^{\mathrm{b}}\right)$ dans la zone d'étude où les eaux de puits sont utilisées à des fins domestiques. 


\section{Conclusion}

Cette étude met en évidence l'état de contamination bactériologique des eaux de puits traditionnels du sixième arrondissement de Cotonou par les salmonelles et les vibrions non-O1/non-O139. Les résultats obtenus montrent que les eaux de puits analysées sont plus exposées à la pollution en saison pluvieuse Cependant, le taux de puits contenant les vibrions non cholériques est plus élevé pendant la petite saison sèche. La présence de vibrions est liée d'une part aux conditions d'hygiène, de l'assainissement dans les quartiers d'étude et d'autre part à la proximité des plans d'eau par rapport aux zones d'étude. La présence des deux germes pathogènes dans les eaux de puits expose la population consommatrice à divers risques sanitaires d'origine hydrique dont les gastro-entérites et les maladies diarrhéiques. Ces eaux sont impropres à la consommation sans aucun traitement. Face à cette situation, il faut sensibiliser la population à l'hygiène du cadre de vie, mettre en place un système de surveillance et de désinfection régulière des eaux de puits et enfin faciliter l'accès à l'eau de distribution publique.

\section{References:}

1. Agassounon Djikpo Tchibozo M., Anani K.T., Ameyapoh V., Toukourou F., De Souza C., Gbeassor M. (2001). Evaluation de la qualité hygiénique de six plantes médicinales et des phytomédicaments traditionnels Pharm. Méd. Trad. Afr. Vol 11 : 83-92.

2. Ali M., Nelson A .R ., Lopez A.L., Sack D.A. (2015). Updated global burden of cholera in endemic countries. PLoS. Negl .Trop. Dis. 9 (6) : 3832

3. Aubry P. (2013). Les Salmonelloses. Médecine Tropicale, 6p.

4. Aubry P., Gaüzère B-A. (2012). Les maladies liées à l'eau. Médecine Tropicale, $7 \mathrm{p}$.

5. Benajiba M.H., Saoud Y., Lamribah A., Ahrikat M., Amajoud N., Ouled-Zian O. (2013). Evaluation de la qualité microbienne des eaux de la nappe phréatique de Martil au Maroc. Revue des Sciences de l'Eau /Journal of Water Science, 26 (3) : 223-233.

6. Blais M. A., Cote C., Villemur R., Généreux M., Cantin P. et Villion M. (2015). Revue des méthodes de détermination de sources de contamination fécale de l'eau. Rapport présenté au ministère du développement durable, de l'environnement et de la lutte contre les changements climatiques, Canada, 39p.

7. Bourgeois C.M. et Leveau J.Y. (1991). Technique d'analyse et contrôle dans les industries agroalimentaires. Contrôle microbiologique. Tome 3 : 327-334.

8. Bras A., Emmanuel E., Obiscon L., Brasseur P., Pape J.W., Raccurt C.P. (2007). Evaluation du risquebiologique dû à Cryptosporidium sp. 
présent dans l'eau de boisson à Port-au-Prince, Haïti. Environnement Risques et Santé. 6: 355-364.

9. Capo A. (2008). Urbanisation et risques naturels : Cas de la ville de Cotonou en République du Bénin. Mémoire de travail de fin d'études en vue de l'obtention du Diplôme d'Ingénieur de l'ESGT, 58p.

10. Carter D., Merson M.H., Spira W.M. (1981). Choléra et autres diarrhées associées à des vibrions, Bulletin de l'Organisation Mondiale de la Santé, 59 (1) : 27-52.

11. CHOLCLIM (2006). Quantification des risques d'émergence d'épidémies à choléra dans le bassin méditerranéen en relation avec le changement climatique, Rapport final, $79 \mathrm{p}$.

12. Colwell R. R., Huq A. (1994). Environmental reservoir of Vibrio cholerae. The causative agent of cholera. Ann. N. Y. Acad. Sci. 740, 44-54.

13. Decret béninois béninois (2001). Décret №2001-94 du 20 février 2001 fixant les normes de qualité de l'eau potable en République du Bénin, $11 \mathrm{p}$.

14. Degbey C., Makoutodé M., Agueh V., Dramaix M., de Brouwer C. (2011). Facteurs associés à la qualité de l'eau de puits et prévalence des maladies hydriques dans la commune d'Abomey-Calavi (Bénin). Santé (21) : 47-55.

15. Dongo K., Kouamé F.K., Koné B., Biém J., Tanner M. et Cissé G. (2008). Situation de l'environnement sanitaire des quartiers défavorisés dans le tissu urbain de Yopougon à Abidjan, Côte d'Ivoire. Revue en sciences de l'environnement. 8 (3)12.

16. Dovonou F., Aïna M.A., Alassane M.B. (2011). Pollution physicochimique et bactériologique d'un écosystème aquatique et ses risques écotoxicologiques : Cas du lac Nokoué au Sud Benin. International Journal of Biologicaland Chemical Sciences. 5: 1590-1602.

17. Dutta D., Chowdhury G., Pazhani G.P., Guin S., Dutta S., Ghosh S., Rajendran K., Nandy R.K., Mukhopadhyay A.K., Bhattacharya M.K., Mitra U., Takeda Y., Nair G.B., Ramamurthy T. (2013). Vibrio cholera non-O1, non-O139 serogroups and cholera-like diarrhea, Kolkata, India. Emerg Infect Dis19: 464-467.

18. Fournier J.M., Quilici M.L. (2002). Infections à vibrions non cholériques. EncyclMédChir, Ed. Scientif. Méd.Elsevier, Paris, Maladies infectieuses. 8-026-F-15, 7 p.

19. Gruzdev N., Pinto R., Sela S. (2011). Effect of Desiccation on Tolerance of Salmonella enteric to Multiple Stresses. Applied and environmental microbiology, 77(5):1667-1673.

20. Habimana O., Nesse L. L., Moretro T., Berg K., Heir E., Vestby L. K., Langsrud S. (2014). The persistence of Salmonella following 
desiccation under feed processing environmental conditions: a subject of relevance. Letters in Applied Microbiology. 59 (5) :464-470.

21. Haijoubie H., Benyahya F., Bendahou A., Essadqui F. Z., El Behhari M., El MamouneA .F. , GhailaniN.N. , MechitaM.B. , Barakat A., 2017. Etude de la qualité bactériologique de l'eau utilisée dans l'industrie agroalimentaire dans le Nord du Maroc. The Pan. African. Medical Journal (26): 13.

22. Hernandez J. Fr. (1989). Etude quantitative et qualitative des vibrions halophiles dans les eaux marines et estuariennes du nord - Pas de calais, Institut pasteur de Lille, Service eaux-environnement, Contrat ifremer 87/6/421/050, 54p.

23. Hervio-Heath D., Colwell R. R., Derrien A., Robert-Pillot A., Fournier J. M. and Pommepuy M. (2002) Occurrence of pathogenic vibrios in coastal areas of France. Journal of Applied Microbiology (92) : 11231135.

24. Hounsounou E., Agassounon Djikpo Tchibozo M., Adjagodo A., Agbossou E., (2014). Etat de l'hygiène et de l'assainissement dans quelques quartiers déshérites de Cotonou (Bénin) pour l'éducation de la population. Rev. Spe. Jour. Sci. FLASH.4 (9) : 171-181.

25. Hounsounou E., Agassounon Djikpo Tchibozo M., Kelomè N.C., Vissin E.W., Mensah G.A., Agbossou E., (2016 ${ }^{\mathrm{a}}$ ). Pollution des eaux à usages domestiques dans les milieux urbains défavorisés des pays en développement : Synthèse bibliographique. International Journal of Biologicaland Chemical Sciences. 10(5) : 2392-2412.

26. Hounsounou E.O., Agassounon Djikpo Tchibozo M., VlavonouZannou M., Vissin E.W., Kelomè N.C., Mensah G.A., et Agbossou E. $\left(2016^{b}\right)$. Pollution fécale des eaux de puits à usages domestiques et risques sanitaires dans le sixième arrondissement de Cotonou au SudBénin. Actes de Colloque en hommage au Professeur Michel Boko, UAC, Bénin : 242-252.

27. Hounsounou E.O., Agassounon Djikpo Tchibozo M., Ayi-Fanou L.,Kelome N. C.,Vissin E.W., Vlavonou-Zannou M ., Mensah G.A., Agbossou E. (2017). Indicateurs de pollution bactériologique des eaux de puits à usages domestiques et risques sanitaires dans le sixième arrondissement de Cotonou au sud-Bénin, Afrique SCIENCE 13(3) :425 - 434.

28. Kahoul M., Touhami M. (2014). Evaluation de la qualité physicochimique des eaux de consommation de la ville d'Annaba(Algérie). Larhyss journal. $\mathrm{n}^{\circ} 19,129-138$.

29. Makoutodé M., Diallo F., Mongbo V., Guévart E., Bazira L. (2010). La riposte à l'épidémie de choléra de 2008 à Cotonou (Bénin). Santé publique. 22 : 425-35. 
30. Ministère de la Santé (MSP), 2008-2012. Annuaire des statistiques sanitaires, 210p.

31. Momba M.N.B., Malakate V.K., Theron J. (2006). Abundance of pathogenic Escherichia coli, Salmonella typhimurium andVibrio cholera in Nkonkobe drinking water sources, J. Water Health, Vol.4, Issue 3, 289-296.

32. Odoulami L. (2009). La problématique de l'eau potable et la santé humaine dans la ville de Cotonou (République du Bénin). Thèse de Doctorat, Université d'Abomey-Calavi, Géographie et Gestion de l'Environnement, $230 \mathrm{p}$.

33. OMS (Organisation mondiale de la santé), (1997). Guideline for Drinking Water Quality 2nd Surveillance and Control of Community Supplies, Geneva. Volume 3,

34. OMS (Organisation mondiale de la santé), (2004). Lien entre l'eau, l'assainissement, l'hygiène et la santé, faits et chiffres, $2 \mathrm{p}$.

35. OMS/UNICEF (2007). Atteindre l'OMD relatif à l'eau potable et à l'assainissement le défi urbain et rural de la décennie. Programme Conjoint OMS/UNICEF de Surveillance de l'Approvisionnement en Eau et de 1'Assainissement, $48 \mathrm{p}$.

36. Quilici M-L. (2011). Le diagnostic bactériologique du choléra, Revue Francophone des Laboratoires - n431 : 29-43.

37. Rodier J., Legube B., Merlet N. (2009). L'Analyse de l'eau, 9 èmeédition DUNOD, 749-775.

38. Roszak D.B., Colwell R. R. (1987). Survival strategies of bacteria in the natural environment. Microbiol. Rev. (51): 365-379.

39. Sudre B., Legoff N. (2011). Mode opératoire : Recherche de Vibrio cholerae dans l'environnement hydrique, $28 \mathrm{p}$.

40. Sy I., Koita M., Traoré D., Keita M., Lo B., Tanne M, Cissé G. (2011). Vulnérabilité sanitaire et environnementale dans les quartiers défavorisés de Nouakchott (Mauritanie) : analyse des conditions d'émergence et de développement des maladies en milieu urbain sahélien. Revue en sciences de l'environnement. 11 (2) : 17. 